\title{
Prevalence of tobacco smoking and association with other unhealthy lifestyle risk factors in the general population of Greece: Results from the EMENO study
}

\author{
Maria Gangadi', Natasa Kalpourtzi ${ }^{2}$, Magda Gavana $^{3}$, Apostolos Vantarakis ${ }^{4}$, Gregory Chlouverakis ${ }^{5}$, Christos Had- \\ jichristodoulou ${ }^{6}$, Gregory Trypsianis ${ }^{7}$, Paraskevi V. Voulgari ${ }^{8}$, Yannis Alamanos ${ }^{9}$, Argiro Karakosta ${ }^{2}$, Giota Touloumi2 ${ }^{2 *}$ \\ Anna Karakatsani ${ }^{*}$
}

\begin{abstract}
INTRODUction The EMENO (National Morbidity and Risk Factors) survey is one of the first and most representative population-based surveys in Greece due to its study design and sampling procedure. We aimed to estimate the prevalence of smoking, secondhand smoking (SHS) and their potential associations with other socioeconomic and unhealthy lifestyle risk factors. METHODS EMENO is a cross-sectional health status survey conducted in Greece from May 2013 to June 2016. The survey was performed using faceto-face interviews and enrolled 6006 adults. Data were collected through questionnaires administered by trained interviewers. Current smoking (CS) and SHS were based on self-reporting. Analysis accounted for study design. RESULTS Information on smoking was available for 5862 individuals (97.6\%). Overall, $37.8 \%$ were current and $16.1 \%$ former smokers. More males (44.3\%) than females (31.6\%) were current smokers. CS increased during adulthood and declined sharply in the elderly $(\mathrm{p}<0.001)$. Smoking initiation by the age of 17 years was reported by $48.7 \%$ of males and $36.2 \%$ of females. Multivariable analysis showed that higher alcohol consumption ( $>7$ glasses/ week, $\mathrm{OR}=2.52 ; 95 \% \mathrm{CI}: 1.97-3.23)$ and lower education level in men were positively associated with ever smoking. Moreover, women aged $>35$ years and respondents with low adherence to the Mediterranean diet (MD) (high/ low, OR=0.35; 95\% CI: 0.21-0.58) had higher odds to be current smokers than former smokers. Finally, the overall prevalence of exposure to SHS at work, home and public places was $38.8 \%, 30 \%$ and $44.6 \%$, respectively. CONCLUSIONS Unhealthy lifestyles of smokers, increased rates of CS in vulnerable groups, such as females and young adults, and early age of smoking initiation constitute alarming public health issues in Greece.
\end{abstract}

\section{AFFILIATION}

1 2nd Pulmonary Department, 'Attikon' University Hospital, Medical School, National and Kapodistrian University of Athens, Athens, Greece

2 Department of Hygiene, Epidemiology and Medical Statistics, School of Medicine, National and Kapodistrian University of Athens, Athens, Greece

3 Department of Primary Care, General Practice and Health Services Research, Medical School, Aristotle University of Thessaloniki, Thessaloniki, Greece 4 Environmental Microbiology Unit, Department of Public Health, Medical School, University of Patras, Patra, Greece 5 Division of Biostatistics, School of Medicine, University of Heraklion, Heraklion, Greece

6 Department of Hygiene and Epidemiology, Medical Faculty, University of Thessaly, Larissa, Greece

7 Department of Medical Statistics, Medical Faculty, Democritus University of Thrace, Alexandroupolis, Greece 8 Rheumatology Clinic, Department of Internal Medicine, Medical School, University of Ioannina, Ioannina, Greece 9 Institute of Epidemiology Preventive Medicine and Public Health, Corfu, Greece ${ }^{*}$ Contributed equally

\section{CORRESPONDENCE TO}

Maria Gangadi. 2nd Pulmonary Medicine Department, "Attikon" University Hospital, Medical School, National and Kapodistrian University of Athens, 1 Rimini street, 12462 Haidari, Athens, Greece. E-mail: gagadim@yahoo.gr ORCID ID: https://orcid. org/0000-0001-7968-4895

\section{KEYWORDS}

smoking prevalence, lifestyle risk factors, alcohol consumption, BMI, Mediterranean diet, Greece

Received: 30 December 2020

Revised: 23 June 2021

Accepted: 15 July 2021 


\section{INTRODUCTION}

Tobacco is a unique product in the sense that even though smokers use it exactly as promoted by manufacturers, many of the smokers get ill or die because of this use ${ }^{1}$. Studies have shown that high blood pressure and smoking were the two leading risk factors of early death and disability worldwide in $2015^{2}$. The World Health Organization (WHO) stated that smoking and smokeless tobacco use accounted for 6 million deaths globally per year, with 0.6 million due to the effects of secondhand smoking (SHS) ${ }^{1}$. Moreover, tobacco use is one of the four modifiable behavioral risk factors of non-communicable diseases $(\mathrm{NCDs})$, along with physical inactivity, harmful alcohol use, and unhealthy diet. Based on the theory that negative health behaviors may cluster together ${ }^{3}$, smokers should have a higher probability of following an unhealthy lifestyle model. However, there are few studies to prove it ${ }^{4}$.

Smoking prevalence in Greece ranks highest among all the Organization for Economic Cooperation and Development (OECD) Countries ${ }^{5}$ and among all European countries according to Eurobarometer surveys ${ }^{6}$. Considering that every year more than 22700 deaths $^{7}$ and $8.9 \%$ of the total hospital admissions in Greece can be attributed to smoking-related diseases ${ }^{8}$, continuous monitoring of tobacco smoking is absolutely necessary. Since 2006, studies on tobacco smoking prevalence in Greece have been conducted demonstrating high rates of use $^{9}$, despite an enhanced anti-tobacco campaign.

The last estimate of smoking prevalence in Greece was gathered by the Global Adult Tobacco Survey (GATS) in 2013 and revealed a $38.2 \%$ prevalence rate for smoking and high rates of SHS ${ }^{10}$. GATS was also used in several countries using a standard process and protocol to collect household tobacco data. The intent of this initiative was to generate internationally comparable data and to attempt implementing the MPOWER package of six proven policies (Monitor, Protect, Offer, Warn, Enforce, Raise) proposed by $\mathrm{WHO}^{10}$. To the best of our knowledge, there are not any studies in Greece associating smoking with other behavioral risk factors for NCDs.

During 2014-2016, a door-to-door cross-sectional health examination survey was conducted in Greece. The primary objectives of the Greek Health
Examination Survey EMENO (National Survey of Morbidity and Risk Factors) were to describe morbidity (focusing on cardiovascular and respiratory diseases) and related risk factors in a random sample of adults representative of the general population. Taking advantage of the EMENO study data, we aimed to: 1) provide a more recent, nationally representative, estimate of the tobacco smoking and SHS prevalence in Greece; 2) investigate the association of being ever smoker with other lifestyle factors; and 3) compare current with former smokers in an effort to see whether smoking cessation is associated with changes to general lifestyle.

\section{METHODS}

\section{Study design}

EMENO, as mentioned above, is a cross-sectional health status survey conducted in Greece from May 2013 to June 2016 (field study conducted during 2014-2016). Ethics approval and consent to participate in the EMENO study was approved by the Ethics and Deontology Committee of the National and Kapodistrian University of Athens (Date: 8 November 2012, Protocol number: 1742) and by the Hellenic Data Protection Authority (Date: 7 December 2012, Protocol number: GN/EX/1069-1/07-12-2012). A modified version of the informed consent form (ICF) was approved by the Ethics and Deontology Committee of the National and Kapodistrian University of Athens (Date: 6 March 2013, Protocol number: 6315).

The survey was conducted by 'door-to-door' interviews using a Computer Assisted Personal Interview (CAPI) and augmented with data and physical examinations performed by trained personnel (reviewers and physicians), in a randomly selected sample representative of the non-institutionalized adult ( $\geq 18$ years) population of Greece. The detailed EMENO study protocol has been described elsewhere ${ }^{11}$. The survey sample consisted of 6006 individuals aged $\geq 18$ years and covered urban (>10000 inhabitants), semi-urban (2000-9999 inhabitants) and rural areas $(<2000$ inhabitants) of the country. The overall response rate to interviews was $72 \%$.

\section{Outcomes of interest}

Self-reported smoking.

Participants who responded that they currently smoke 
were classified as 'current smokers'. Those who smoked any time in the past but had stopped were classified as 'former smokers', whilst 'never smokers' included those who had never smoked.

\section{Secondhand smoking (SHS)}

SHS referred to smoking exposure at home, workplaces, public places and transportation during the last six months. The respondents of each category were categorized into four sub-groups according to the hours of exposure: Never; $<1$ h/day; $1-5$ h/day; and $>5 \mathrm{~h}$ /day.

\section{Tobacco consumption}

Current and former smokers were also asked to report the number of tobacco products (manufactured and hand-rolled cigarettes, cigars and cigarillos, tobacco pipe, and other) that they smoked on average per day and for how many years. Pack-years were calculated only for manufactured and hand-rolled cigarettes. Moreover, they reported the age at which they started smoking regularly and their responses were grouped into four age groups $(10-15,16-17,18-22,>22$ years).

\section{Covariates}

\section{Sociodemographic characteristics}

All individuals were asked to report their gender, age, level of education, place of residence and partnership status. Age was recoded as a categorical variable: 18 $34,35-49,50-65$, and $>65$ years. Education level was categorized in three groups based on the individuals' highest level of completed education: Primary, Secondary (Junior and/or Senior high school)-Post secondary (2 years of Vocational Education), and Tertiary (Technological Institutions and Universities). Employment status was recoded as a dichotomous variable: employed, not employed.

The International Standard Classification of Occupations (ISCO-88) scale was used to record the occupational group of each respondent. These job categories were further grouped in low and high skilled 'blue-collar' and 'white-collar' jobs, as proposed by the European Foundation for the Improvement of Living and Working Condition. Based on this classification a 'blue-collar worker' is a member of the working class who performs manual labor (ISCO-88: 6-9) and a 'white-collar worker' is a salaried professional, typically referring to general office workers and management (ISCO-88: 1-5) ${ }^{12}$.

Participants' place of residence was categorized into three groups: urban, semi-urban and rural areas; whereas their partnership status was dichotomized as either single or living with a partner.

\section{Body Mass Index (BMI)}

The height and weight, after being measured using standardized equipment, were used to calculate the BMI for each individual and the WHO cut-off points for overweight ( $\left.\geq 25 \mathrm{~kg} / \mathrm{m}^{2}\right)$ and obesity $\left(\geq 30 \mathrm{~kg} / \mathrm{m}^{2}\right)$ were applied for the classification of participants as normal weight, overweight, or obese.

\section{Physical activity}

Physical activity was measured using the International Activity Questionnaire (IPAQ ) ${ }^{13}$ the reliability of which has been previously evaluated in the Greek population ${ }^{14}$. Therefore, individuals were categorized into three activity levels: low, moderate, or high, as suggested ${ }^{14}$.

\section{Alcohol consumption}

Participants were asked about the number of glasses of all alcoholic beverages (beer, wine, liquor) they consumed during the previous week and their responses were grouped into three categories $(0,1-7$, $>7$ glasses/week).

\section{Mediterranean diet}

The level of adherence to Mediterranean diet (MD) was measured by the Mediterranean diet adherence screening (MEDAS) questionnaire ${ }^{15}$. This is a 14 -item questionnaire (score: $0-14$ ) that has been validated and used in several countries including Spain ${ }^{15}$, Germany $^{16}$, as well as Greece ${ }^{17}$. Participants were classified as having low, medium, or high adherence to MD if their score was $0-4,5-9$, and 10-14, respectively.

\section{Statistical analysis}

All EMENO participants with known smoking status (current, former, or never smokers) were included in the analysis. Sampling weights, being the reciprocal of the selection probabilities, were applied to adjust for the sampling design; sampling weights were multiplied by a correction factor to adjust for the discrepancies in 
the gender, age, and geographical area distribution of the sample from the corresponding one of the adult ( $\geq 18$ years) general population (based on the 2011 census, provided by the National Statistics Agency).

Weighted means and standard deviations for normally distributed, medians and interquartile range (IQR) for non-normally distributed continuous variables and weighted percentages for categorical variables were provided. Weighted multivariable logistic regression models were fitted to investigate factors associated with ever smoking (ever vs never) or being current smoker (current vs former smoker). Demographic and socioeconomic variables (age, gender, educational level, partnership status, job status, 'blue or white collar' classification, place of residence), lifestyle factors (alcohol consumption, Mediterranean diet, physical activity) and BMI were investigated as potential covariates. Backward elimination procedure was implemented to select the statistically significant covariates and possible interaction effects were also investigated.
In non-binary categorical variables, categories were collapsed if there were no indications for significant differences in the estimated ORs based on the Wald test.

\section{RESULTS}

\section{General features of smoking prevalence in}

\section{Greece}

Out of 6006 individuals participating in the survey, 13 had missing values for age and gender and 131 (2.2\%) for smoking status, yielding a total of 5862 study participants. The characteristics of the study population, expressed also as weighted averages, are presented in Table 1.

The majority of the study population $(62.7 \%)$ was living in urban areas, $47.1 \%$ had finished at least secondary school and $40 \%$ had less than 900 euros monthly family income. Forty-three percent were non-drinkers and $42.1 \%$ moderate drinkers, whereas nearly $80 \%$ had moderate adherence to MD (79.5\%) and $71.3 \%$ moderate/high physical activity.

Table 1. Characteristics of the study population, overall and by smoking status in Greece, during 2014-2016 $(\mathrm{N}=5862)$

\begin{tabular}{|c|c|c|c|c|c|}
\hline Characteristics & $\begin{array}{l}\text { Smokers } \\
n(\%)^{a}\end{array}$ & $\begin{array}{l}\text { Former smokers } \\
\qquad n(\%)^{a}\end{array}$ & $\begin{array}{c}\text { Never smokers } \\
n(\%)^{a}\end{array}$ & $\begin{array}{c}\text { Total } \\
n(\%)^{a}\end{array}$ & $p^{b}$ \\
\hline \multicolumn{6}{|l|}{ Gender } \\
\hline Men & $1018(56.9)$ & $742(73.8)$ & $722(32.7)$ & $2482(48.5)$ & \\
\hline Women & $1008(43.1)$ & $290(26.2)$ & $2082(67.3)$ & $3380(51.5)$ & \\
\hline Age (years) & & & & & $<0.001$ \\
\hline $18-34$ & $427(30.8)$ & $76(12.3)$ & $473(26.8)$ & $976(26.0)$ & \\
\hline $35-49$ & $685(35.6)$ & $190(23.3)$ & $571(23.2)$ & $1446(27.9)$ & \\
\hline $50-65$ & $626(23.6)$ & $325(28.4)$ & 700 (18.8) & 1651 (22.2) & \\
\hline$>65$ & $288(10.0)$ & $441(36.0)$ & $1060(31.2)$ & 1789 (24.0) & \\
\hline Degree of urbanization & & & & & $<0.001$ \\
\hline Urban & $1234(66.6)$ & $538(55.9)$ & $1558(61.9)$ & $3330(62.7)$ & \\
\hline Semi-urban & $339(15.0)$ & $194(18.1)$ & $512(17.3)$ & $1045(16.6)$ & \\
\hline Rural & $453(18.4)$ & $300(26.0)$ & $734(20.8)$ & $1387(20.7)$ & \\
\hline Family status & & & & & $<0.001$ \\
\hline Married/cohabitating & $1330(60.6)$ & $803(75.1)$ & $1748(57.5)$ & $3881(61.5)$ & \\
\hline Single & $689(39.0)$ & $226(24.5)$ & $1046(42.1)$ & $1961(38.1)$ & \\
\hline Unknown & $7(0.3)$ & $3(0.4)$ & $10(0.4)$ & $20(0.4)$ & \\
\hline Education level & & & & & $<0.001$ \\
\hline Primary & $517(21.0)$ & 412 (33.9) & 1149 (33.7) & $2078(28.9)$ & \\
\hline Secondary/post-secondary & $1054(55.0)$ & $400(41.4)$ & $1104(42.5)$ & $2558(47.1)$ & \\
\hline Higher education & $442(23.4)$ & $215(24.2)$ & $533(23.2)$ & 1190 (23.4) & \\
\hline Unknown & $13(0.6)$ & $5(0.5)$ & $18(0.6)$ & $36(0.6)$ & \\
\hline
\end{tabular}


Table 1. Continued

\begin{tabular}{|c|c|c|c|c|c|}
\hline Characteristics & $\begin{array}{l}\text { Smokers } \\
n(\%)^{a}\end{array}$ & $\begin{array}{c}\text { Former smokers } \\
\qquad n(\%)^{a}\end{array}$ & $\begin{array}{c}\text { Never smokers } \\
n(\%)^{a}\end{array}$ & $\begin{array}{c}\text { Total } \\
n(\%)^{a}\end{array}$ & $p^{b}$ \\
\hline Unemployed & & & & & $<0.001$ \\
\hline No & 1632 (78.5) & $933(88.7)$ & $2497(87.1)$ & $5062(84.1)$ & \\
\hline Yes & $386(21.1)$ & $96(11.0)$ & $289(12.2)$ & $771(15.4)$ & \\
\hline Unknown & $8(0.4)$ & $3(0.4)$ & $18(0.6)$ & $29(0.5)$ & \\
\hline Job category & & & & & $<0.001$ \\
\hline High skilled white-collar & $489(23.6)$ & $245(25.6)$ & 509 (19.9) & $1243(22.2)$ & \\
\hline Low skilled white-collar & 497 (25.4) & $188(18.6)$ & $445(17.0)$ & 1130 (20.4) & \\
\hline High skilled blue-collar & $308(14.4)$ & $251(22.3)$ & $565(17.5)$ & $1124(17.1)$ & \\
\hline Low skilled blue-collar & $429(20.8)$ & $255(24.6)$ & $620(21.0)$ & $1304(21.5)$ & \\
\hline Unknown & $303(15.9)$ & $93(9.0)$ & $665(24.6)$ & 1061 (18.8) & \\
\hline Monthly family income $(€)$ & & & & & 0.090 \\
\hline$<900$ & $812(40.3)$ & $388(37.4)$ & $1187(41.4)$ & $2387(40.3)$ & \\
\hline $900-1700$ & 579 (28.9) & $323(30.4)$ & $740(27.3)$ & $1642(28.4)$ & \\
\hline$>1700$ & $222(11.0)$ & $124(13.3)$ & $255(10.2)$ & $601(11.0)$ & \\
\hline Unknown & $413(19.8)$ & $197(18.9)$ & $622(21.1)$ & $1232(20.3)$ & \\
\hline Physical activity & & & & & 0.004 \\
\hline Low & $260(12.7)$ & $181(17.0)$ & $434(15.2)$ & $875(14.6)$ & \\
\hline Moderate & $550(27.9)$ & $320(31.3)$ & $788(28.9)$ & $1658(28.9)$ & \\
\hline High & $912(44.4)$ & $383(37.9)$ & $1209(42.3)$ & $2504(42.4)$ & \\
\hline Unknown & $304(15.0)$ & $148(13.8)$ & $373(13.6)$ & $825(14.2)$ & \\
\hline Adherence to Mediterranean diet & & & & & $<0.001$ \\
\hline Low & $208(13.1)$ & $49(5.6)$ & $195(8.6)$ & 452 (9.8) & \\
\hline Moderate & 1595 (76.4) & $848(82.6)$ & $2296(81.0)$ & 4739 (79.5) & \\
\hline High & $130(5.8)$ & $114(9.8)$ & $226(6.9)$ & $470(7.0)$ & \\
\hline Unknown & $93(4.7)$ & $21(2.0)$ & $87(3.5)$ & $201(3.7)$ & \\
\hline Alcohol consumption (glasses/week) & & & & & $<0.001$ \\
\hline 0 & $746(34.1)$ & $340(32.7)$ & $1615(54.1)$ & $2701(43.1)$ & \\
\hline $1-7$ & $888(46.2)$ & $477(45.7)$ & $971(37.5)$ & $2336(42.1)$ & \\
\hline$>7$ & $335(16.6)$ & $199(20.1)$ & $170(6.4)$ & $704(12.5)$ & \\
\hline Unknown & $57(3.1)$ & $16(1.5)$ & $48(2.0)$ & $121(2.4)$ & \\
\hline BMI & & & & & $<0.001$ \\
\hline Normal & $546(28)$ & 143 (15.5) & $524(21.1)$ & 1213 (22.8) & \\
\hline Overweight & $600(28.5)$ & $346(33.4)$ & $812(28.2)$ & 1758 (29.2) & \\
\hline Obese & $478(21.7)$ & $368(33.4)$ & 844 (26.2) & 1690 (25.6) & \\
\hline Unknown & 402 (21.8) & $175(17.6)$ & $624(24.5)$ & 1201 (22.4) & \\
\hline
\end{tabular}

a Weighted percentages. b The p-values were calculated using Rao-Scott chi-squared test excluding missing values.

Regarding BMI, $29.2 \%$ of the study population were overweight. The overall prevalence of current smoking was $37.8 \%$ (95\% CI: $36.3-39.3)$ and of former smoking 16.1\% (95\% CI: 15.1-17.2) (Table $1)$. Descriptive characteristics by smoking status are also shown in Table 1, with the distribution of all presenting factors being different among smoking groups. There were $98(3.2 \%)$ participants with missing data for pack-year consumption of manufactured and hand-rolled cigarettes. Among those with available information, the median number of pack-years (cigarettes and hand-roll cigarettes) of ever smokers was 18.6 (IQR: 7.5-36.5). Boxed cigarettes are the tobacco product most used among 
Figure 1. Prevalence of current and former smoking by age and gender (a) and mean age at onset of smoking by gender (b) in Greece during 2014-2016
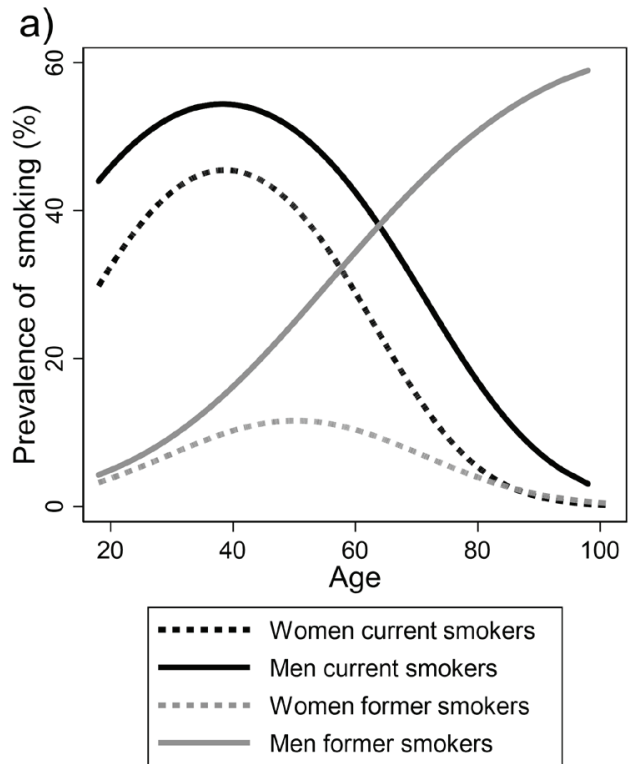

smokers (91\% of all tobacco products) (data not shown).

Current smoking is prevalent during adulthood (18-29 years: $44.6 \% ; 30-39$ years: $47 \% ; 40-49$ years: $48.1 \%$; 50-59 years: $42.9 \%$; $<0.001)$, but declines sharply in the elderly (60-69 years: $29.4 \%$; >70 years: $12.7 \%$; $\mathrm{p}<0.001)$. This generational difference is much more evident in women (Figure 1a). Among former smokers, the findings were exactly the reverse (18-29 years: 6.4\%; 30-39 years: $11.3 \%$; $40-$ 49 years: $14.3 \% ; 50-59$ years: $18.7 \%$; $60-69$ years: $26.3 \%$; $>70$ years: $23.11 \%$; $<0.001)$. Both genders had high prevalence of current smoking, but men were more likely than women to be current $(44.3 \%$ vs $31.6 \%, \mathrm{p}<0.001$ ) (Figure 1a) and heavier smokers [median pack-years (IQR): 22.5 (10-45) vs 13.5 (5.5-25), p<0.001] (results not shown). Conversely, women were less likely to be former smokers $(8.2 \%$ vs $24.5 \%$, p <0.001) than men (Figure 1a).

For 49 participants ( $1.6 \%$ of ever smokers), the age at initiation of tobacco smoking was unknown. Among those with available information, the median age of daily smoking initiation for ever smokers (current and former smokers) was 18 years for women (IQR: 16-21) and 18 years for men (IQR: 15-20). It is remarkable that one in two males $(48.7 \%)$ and one in three females $(36.2 \%)$ reported starting smoking before the age of 17

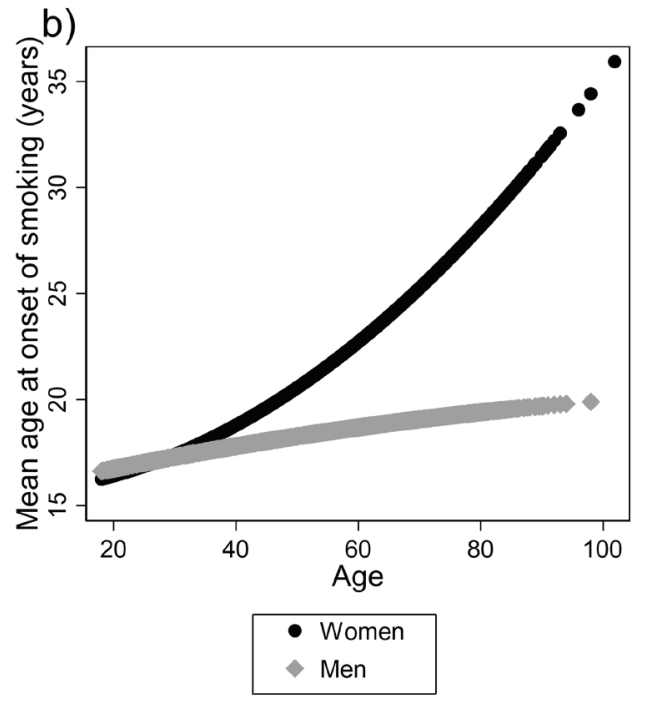

years. Moreover, $31.3 \%$ of ever smokers aged 18-29 years reported smoking initiation at age $<15$ years (results not shown). However, the crucial ages for smoking initiation in both genders appears to be the four-year period between 18 and 22, during which a substantial proportion take up smoking ( $41.9 \%$ of males and $44.5 \%$ of females). Finally, there seems to be generational differences regarding the age of smoking initiation for both sexes. Younger individuals reported to start smoking earlier than older ones. This difference is particularly evident in older women (Figure 1b).

\section{Multivariable analysis to assess the factors associated with being ever smoker}

All factors presented in Table 1 were assessed for their independent association with ever smoking. Results from multivariable logistic regression showed that among all investigated factors, only alcohol consumption and unemployment status were independently associated with ever being a smoker (Table 2). More specifically, the heavier the alcohol consumption the higher were the odds of ever smoking; compared to non-drinkers, those who were drinking 1-7 glasses per week had $41 \%$ higher odds, and those who were drinking $>7$ glasses had $152 \%$ higher odds of being ever smokers.

Additionally, unemployed participants had 
$42 \%$ higher odds of being ever smokers compared to employed participants (Table 2). Significant

Table 2. Factors associated with being ever smoker in Greece during 2014-2016: Results from multivariable logistic model ( $N=5862)$

\begin{tabular}{|c|c|c|}
\hline Characteristics & OR $(95 \% \mathrm{CI})$ & $p$ \\
\hline \multicolumn{3}{|c|}{ Alcohol consumption (glasses/week) } \\
\hline $1-7$ & $1.41(1.22-1.64)$ & $<0.001$ \\
\hline$>7$ & $2.52(1.97-3.23)$ & 0.001 \\
\hline Unemployed/employed & $1.42(1.16-1.73)$ & 0.001 \\
\hline \multicolumn{3}{|l|}{ Age (18-34.9 years) } \\
\hline \multicolumn{3}{|l|}{ Women } \\
\hline Secondary/Higher vs Primary & $0.84(0.43-1.66)$ & 0.614 \\
\hline \multicolumn{3}{|l|}{ Men } \\
\hline Secondary/Higher vs Primary & $0.44(0.23-0.84)$ & 0.013 \\
\hline \multicolumn{3}{|l|}{ Primary education } \\
\hline Women/Men & $0.37(0.23-0.59)$ & $<0.001$ \\
\hline \multicolumn{3}{|l|}{ Secondary/Higher education } \\
\hline Women/Men & $0.71(0.54-0.94)$ & 0.015 \\
\hline \multicolumn{3}{|l|}{ Age (35-49.9 years) } \\
\hline \multicolumn{3}{|l|}{ Women } \\
\hline Secondary/Higher vs Primary & $1.28(0.85-1.92)$ & 0.243 \\
\hline \multicolumn{3}{|l|}{ Men } \\
\hline Secondary/Higher vs Primary & $0.67(0.44-1.03)$ & 0.066 \\
\hline \multicolumn{3}{|l|}{ Primary education } \\
\hline Women/Men & $0.35(0.23-0.53)$ & $<0.001$ \\
\hline \multicolumn{3}{|l|}{ Secondary/Higher education } \\
\hline Women/Men & $0.67(0.52-0.87)$ & 0.002 \\
\hline \multicolumn{3}{|l|}{ Age (50-64.9 years) } \\
\hline \multicolumn{3}{|l|}{ Women } \\
\hline Secondary/Higher vs Primary & $1.00(0.75-1.34)$ & 0.991 \\
\hline \multicolumn{3}{|l|}{ Men } \\
\hline Secondary/Higher vs Primary & $0.52(0.35-0.79)$ & 0.002 \\
\hline \multicolumn{3}{|l|}{ Primary education } \\
\hline Women/Men & $0.17(0.12-0.26)$ & $<0.001$ \\
\hline \multicolumn{3}{|l|}{ Secondary/Higher education } \\
\hline Women/Men & $0.33(0.25-0.43)$ & $<0.001$ \\
\hline \multicolumn{3}{|l|}{ Age ( $\geq 65$ years) } \\
\hline \multicolumn{3}{|l|}{ Women } \\
\hline Secondary/Higher vs Primary & $2.19(1.57-3.06)$ & $<0.001$ \\
\hline \multicolumn{3}{|l|}{ Men } \\
\hline Secondary/Higher vs Primary & $1.15(0.84-1.57)$ & 0.373 \\
\hline \multicolumn{3}{|l|}{ Primary education } \\
\hline Women/Men & $0.09(0.07-0.11)$ & $<0.001$ \\
\hline \multicolumn{3}{|l|}{ Secondary/Higher education } \\
\hline Women/Men & $0.16(0.11-0.23)$ & $<0.001$ \\
\hline
\end{tabular}

interactions were found between age and gender $(\mathrm{p}<0.001)$, age and educational level $(\mathrm{p}=0.001)$, and gender and educational level $(p=0.001)$. As there were no significant differences between those who had completed secondary and those with higher education across gender and age groups, these two groups were combined and compared with those who had only primary education. Educational level was not significantly associated with the odds of ever smoking among women aged 18-65 years, whereas for women aged $>65$ years having secondary or higher educational level compared to having a primary education level was associated with significantly higher odds of ever being a smoker (OR=2.19; 95\% CI: 1.57-3.06) (Table 2). On the other hand, among men, having a higher educational level was associated with lower odds of ever being a smoker for all age groups $(\mathrm{OR}=0.44$; $95 \%$ CI: $0.24-$ 0.84 for those aged $18-34.9$ years; OR $=0.67 ; 95 \%$ CI: $0.44-1.03$ for those aged 35-49.9 years, and $\mathrm{OR}=0.52 ; 95 \%$ CI: $0.35-0.79$ for those aged $50-64.9$ years), apart from those being $\geq 65$ years.

Females were less likely to have ever been smokers compared to males with the difference between the two genders being more pronounced for those who had only primary education compared to those having higher education as well as for those aged $>50$ years (Table 2 ).

\section{Multivariable analysis to compare current with former smokers}

To investigate factors associated with giving up smoking, we restricted the analysis to those who had ever been smokers and compared current with former smokers. Current smokers reported lower levels of adherence to MD [compared to those with low adherence levels, the adjusted ORs (95\% CI) of being current smoker were 0.55 (95\% CI: 0.360.83 ) and 0.35 (95\% CI: 0.21-0.58) for those with moderate and high adherence levels, respectively]. Furthermore, they were less likely to be either overweight (OR=0.71; 95\% CI: 0.54-0.95) or obese $(\mathrm{OR}=0.60 ; 95 \%$ CI: $0.45-0.80)$, and more likely to be single $(\mathrm{OR}=1.47 ; 95 \%$ CI: 1.15-1.88) compared to former smokers (Table 3). Two significant interactions, one between age group and educational level $(p=0.019)$ and one between gender and age group $(p=0.024)$, were found. For those aged $<50$ 
Table 3. Factors associated with being current smoker among ever smokers in Greece during 2014-2016: Results from multivariable logistic model $(\mathrm{N}=5862)$

\begin{tabular}{|c|c|c|}
\hline Variable & OR $(95 \%$ CI $)$ & $p$ \\
\hline \multicolumn{3}{|c|}{ Adherence to Mediterranean diet } \\
\hline Medium/low & $0.55(0.36-0.83)$ & 0.004 \\
\hline High/low & $0.35(0.21-0.58)$ & $<0.001$ \\
\hline Single/living with a partner & $1.47(1.15-1.88)$ & 0.002 \\
\hline \multicolumn{3}{|l|}{ BMI } \\
\hline Overweight/normal & $0.71(0.54-0.95)$ & 0.020 \\
\hline Obese/normal & $0.60(0.45-0.80)$ & $<0.001$ \\
\hline \multicolumn{3}{|l|}{ Age (18-34.9 years) } \\
\hline Women/Men & $1.11(0.61-2.03)$ & 0.731 \\
\hline Secondary/Higher vs Primary & $0.17(0.02-1.49)$ & 0.108 \\
\hline \multicolumn{3}{|l|}{ Age (35-49.9 years) } \\
\hline Women/Men & $1.66(1.14-2.44)$ & 0.009 \\
\hline Secondary/Higher vs Primary & $0.48(0.24-0.97)$ & 0.041 \\
\hline \multicolumn{3}{|l|}{ Age (50-64.9 years) } \\
\hline Women/Men & $2.00(1.39-2.87)$ & $<0.001$ \\
\hline Secondary/Higher vs Primary & $1.12(0.78-1.61)$ & 0.524 \\
\hline \multicolumn{3}{|l|}{ Age ( $\geq 65$ years) } \\
\hline Women/Men & $3.35(2.11-5.32)$ & $<0.001$ \\
\hline Secondary/Higher vs Primary & $1.42(0.98-2.06)$ & 0.066 \\
\hline
\end{tabular}

years, those having secondary or higher education were associated with lower odds of being current than former smokers compared to participants having only primary education. There were no significant differences from the odds of being current smokers in relation to their educational level for those aged $>50$ years (Table 3 ). The gender effect differed across age groups, but it was the same across educational levels within the same age group. Whereas there were no significant differences in the odds of being a current smoker between women and men of the younger age group (18-35 years), in the other age groups the older the age, the more likely were women to be current smokers compared to men (Table 3 ). In general, the odds of being current smokers decreased with increasing age, with age differences being more pronounced among men of primary education.

\section{Secondhand smoking in Greece}

In Table 4, information on the prevalence of reported exposure to SHS during the last six months is presented. Overall, $58.3 \%$ of the respondents were exposed to SHS. The prevalence of exposure was

Table 4. Prevalence of exposure to secondhand smoke at work, home and various public places in Greece during 2014-2016

\begin{tabular}{|c|c|c|c|c|}
\hline Secondhand exposure & $\begin{array}{l}\text { Overall } \\
n(\%)\end{array}$ & $\begin{array}{c}\text { Ever } \\
\text { smokers } \\
n(\%)\end{array}$ & $\begin{array}{c}\text { Never } \\
\text { smokers } \\
n(\%)\end{array}$ & $p$ \\
\hline Home (n) & 5736 & 2993 & 2743 & $<0.001$ \\
\hline Never & $4041(70.0)$ & $1791(59.3)$ & $2250(82.4)$ & \\
\hline$<1 \mathrm{~h} /$ day & $700(12.1)$ & $449(14.9)$ & $251(8.9)$ & \\
\hline $1-5 \mathrm{~h} /$ day & $692(12.2)$ & $524(17.6)$ & $168(6)$ & \\
\hline$>5$ h/day & $303(5.7)$ & $229(8.2)$ & $74(2.8)$ & \\
\hline Workplace (n) ${ }^{a}$ & 1920 & 1139 & 781 & $<0.001$ \\
\hline Never & $1226(61.2)$ & $645(54.3)$ & $581(71.5)$ & \\
\hline$<1 \mathrm{~h} /$ day & $276(15.2)$ & $190(17.1)$ & $86(12.4)$ & \\
\hline $1-5$ h/day & $259(14.8)$ & $186(17.6)$ & $73(10.8)$ & \\
\hline$>5$ h/day & $159(8.7)$ & $118(11.1)$ & $41(5.3)$ & \\
\hline Public places and transportation (n) & 5670 & 2948 & 2722 & $<0.001$ \\
\hline Never & $3369(55.4)$ & $1431(45.3)$ & $1938(67.1)$ & \\
\hline$<1$ h/day & 1381 (26.4) & 862 (30.8) & 519 (21.2) & \\
\hline 1-5 h/day & 781 (15.5) & 546 (19.9) & 235 (10.4) & \\
\hline$>5$ h/day & 139 (2.8) & $109(4.0)$ & $30(1.3)$ & \\
\hline Any place (n) & 5732 & 2988 & 2744 & $<0.001$ \\
\hline Yes & 3155 (58.3) & 2056 (71.1) & 1099 (43.5) & \\
\hline
\end{tabular}

a Among those who were employed. 
$38.8 \%$ at work, $30 \%$ at home, and $44.6 \%$ in public places. The majority of the population was exposed to SHS for less than five hours per day regardless of place of exposure. Tobacco ever smokers reported higher percentages of passive smoking either overall $(71.1 \%)$ or in public and working places $(54.7 \%$ and $45.7 \%$, respectively), compared to never smokers (43.5\%, $32.9 \%$, and $28.5 \%$, respectively).

\section{DISCUSSION}

The EMENO study, conducted from 2013 to 2016 in Greece, was able to provide accurate estimates on the prevalence of smoking and on its determinants of health in the general Greek adult population. A rather high prevalence of current smoking (37.8\%) was observed. Interestingly, the prevalence remained stable since 2011 and in line with previous nationwide studies ${ }^{9,10}$, possibly reflecting the unsuccessful implementation of the anti-smoking legislation. Moreover, it is significantly higher compared to the average $(26 \%)$ of other European countries ${ }^{18}$.

Among the determinants of smoking status, gender was a strong independent factor for current smoking. Specifically, males appeared to have a higher prevalence of current smoking and they were heavier smokers compared to females, as is the case in most countries of the world historically ${ }^{18}$. However, the prevalence of current smoking in Greek females (31.6\%) seems significantly high, being in accordance with the results of the European Health Interview Survey (EHIS-2014) in which Greek women ranked second in prevalence of daily smoking cigarettes $(21.3 \%)$ and were the heaviest smokers in Europe (9.4\% smoked $>20$ cigarettes/ day $)^{18}$. Indeed, the results of our study support the alarming increase of smoking in female groups as: 1) The prevalence of current smoking is high in younger women; 2) Girls are starting to smoke at earlier ages; and 3) The percentages of former smokers, especially in the older age groups, are significantly lower in females than in males. This is a multifactorial event and may be due to female vulnerability towards tobacco marketing ${ }^{19}$, as well as concerns about weight gain and female confidence ${ }^{20}$.

Age was also an important predictor of current smoking. More specifically, the prevalence of current smoking was considerably higher during adulthood, but decreased sharply in the elderly. This age-related pattern is similar to observations in other studies ${ }^{10,18}$ and may be due to the increased smoking-related diseases of advanced age. Another worrying result is that one in three young adults started smoking at age $<15$ years, whilst the corresponding European percentage is much lower $(19 \%)^{6}$. All the above point to the urgent need, at least for the local policy makers, to focus on younger ages than teens to reinforce positive attitudes towards adaptation of healthy habits and avoidance of smoking initiation.

Concerning socioeconomic factors, ever smokers have higher odds of being unemployed than nonsmokers, and current smokers have higher odds of being single/not married compared to former smokers. Similarly, in Europe, unemployed individuals and those having financial straits are more often smokers ${ }^{6}$, probably either due to higher levels of psychological distress ${ }^{21}$ or due to specific characteristics of their personality ${ }^{22}$. Regarding the fact that smokers are more often single, a US study concluded that smoking helps to cope with the negative feelings associated with social isolation ${ }^{23}$.

In the present study, there seems to be no clear trend between the educational level and smoking. Various national and international studies present contradicting results due to different methodologies used. The EHIS concluded that people with tertiary education exhibit the lowest levels of daily cigarette smoking $^{18}$. On the other hand, GATS after adjusting for other covariates, found that Greek people of higher educational level are more likely to be current smokers than never smokers ${ }^{10}$. In EMENO, examining the interaction between educational level and age, we found interesting results as younger individuals of higher educational level were more likely to be former smokers. Moreover, higher educational level appears to be clearly protective only for ever tobacco smoking males but not for females. These results, in combination with similar prevalence estimates of smoking among men and women, could be explained by the theory of the 'smoking epidemic'24. This theory explains that there is a 'four-stage model' of smoking trajectory and probably the progression from one stage to the other is slightly different between countries. Based on this model, smoking initiation is earlier in men than women but gradually, from stage 1 to stage 4 , males drop from high smoking rates to low, followed 
by females with a lag of a few decades. Moreover, in this model's trajectory, individuals with higher educational level stop smoking first because they are the first to realize the smoking-related hazard for their health ${ }^{25}$. The 'smoking epidemic' in Greece based on the results of the EMENO study is probably at the beginning of stage 3 , where the smoking rates of females are similar to those of men and at which stage men of higher educational level quit smoking. At the end of this stage (usually lasting 20-30 years), it is expected that women of higher education will also quit smoking. People of primary education level usually stop smoking at the last stage of this epidemic.

Regarding the associations we found with other health indicators, it seems that former smokers have a higher Body Mass Index (BMI) in relation to current smokers. This result highlights one of the main deterrents for smoking cessation that is the fact that former smokers gain weight after smoking cessation ${ }^{26}$. The mechanism of weight gain after giving up smoking is multivariable and includes increased energy expenditure, decreased resting metabolic rate, as well as increased lipoprotein lipase activity ${ }^{27}$.

Concerning dietary habits, there is evidence that current smokers adhere less often to the Mediterranean diet. This is an important observation as it is well known that the Mediterranean diet is rich in vitamins, fiber, and anti-oxidants and there is also evidence that may counterbalance the negative effects of the elevated oxidative stress of smoking ${ }^{28}$. In addition, we observed that ever smokers consumed large quantities of alcohol compared to non-smokers. Many studies demonstrated a significant association between alcohol drinking and tobacco use in different countries ${ }^{29,30}$. Mammas et al. ${ }^{31}$ observed that alcohol use among Greek medical students was higher in smokers than never smokers. However, so far, to the best of our knowledge, there is no study concerning the general population of Greece. Reducing modifiable risk factors for NCDs such as tobacco use, harmful use of alcohol, unhealthy diet, and physical inactivity through creation of healthpromoting environments constitutes some of the 'best byes' proposed by $\mathrm{WHO}^{32}$. Taking action on reducing these factors is important since 41 million people die annually as a result of NCDs and nearly one-third of these deaths occur between the ages of 30 and 70 years $^{32}$. In Greece, the aforementioned risk factors are among the first top 10 risks contributing to increased disability-adjusted life years (DALYs) in 2019. Information retrieved by Eurostat and OECD health database, show $42 \%$ of all deaths can be attributed to these factors, compared to $39 \%$ in the rest of the European Union ${ }^{5}$. Although several studies recorded prevalence rates of smoking and its sociodemographic determinants in Greece ${ }^{9,10}$, coexistence of multiple health risk factors in a smoker's life have not been assessed previously. Our results clearly demonstrate that smokers in Greece have high risk to cluster health-related behaviors, presuming that ultimately their overall health condition is due to the combined effect of multiple risk factors.

When it comes to SHS, the high exposure rates are alarming since it appears that around two out of five people in Greece are exposed to SHS in their workplace and almost half of them in public places and transport. Citing the results of Eurobarometer in 2014, Greece besides being a country with a high prevalence of current smoking, has also one of the highest rates of SHS in public places in Europe. Thus, while the average exposure rate in Europe for entertainment venues and restaurants is $25 \%$ and $12 \%$, respectively, these rates in Greece are $83 \%$ and $72 \%$ with an upward trend ${ }^{6}$. Taking into consideration the WHO statements about high mortality rates worldwide due to tobacco secondhand exposure ${ }^{33}$, our results point to the need to implement in Greece effective prevention policies to minimize it, especially in public places and workplaces.

\section{Future perspectives}

In Greece, the introduction of legislative measures to limit tobacco exposure began in 1952. For more than 60 years, a multitude of laws have been enacted that aimed at both a smoking ban in public places as well as the banning of any form of advertising campaign. However, looking at the high rates of current smoking and SHS exposure levels that are still present, we understand that the existing smoking bans have not been satisfactorily implemented and enforced. It would seem appropriate that a similar study should 
be conducted in Greece, to confirm or disprove our results, in the near future. In this way, the impact on the percentage of both smoking and passive smoking exposure can be ascertained following the enhanced anti-smoking campaign that was initiated in the beginning of 2020. Moreover, based on the findings of the EMENO that smokers were more likely to combine other lifestyle risk factors, (unhealthy diet and harmful alcohol use) it will be interesting to plan future studies on these health-related behaviors and mortality rates, not only nationally but also across Europe.

\section{Strengths and limitations}

The representative sample of the adult Greek population, the satisfactory response rate, the collection of data by trained interviewers, and the low rate of missing values are the strengths of our study and offer the opportunity to draw some generalizable conclusions regarding smoking prevalence and smokers' lifestyle in Greece. The most significant limitation, as with other similar studies, is the self-reported nature of the data collected, which may result in under-reporting of smoking. However, a review study on self-reporting of smoking behavior, concluded that self-reports of smoking were quite accurate ${ }^{34}$. It is worth noting that as our study methodology was quite different from those implemented in other national and international studies on smoking prevalence, our results may not be comparable.

\section{CONCLUSIONS}

The EMENO, in accordance with previous studies, revealed a high prevalence of current and passive smoking among the adult Greek population. Females and younger people seem to be vulnerable groups and the state should focus on creating targeted tobacco programs and policies. Finally, smokers should be informed about the benefits of an overall healthy lifestyle, as it seems that smoking cessation must reflect not only an isolated modification of a specific behavior but also, most importantly, a generally change in an individual's lifestyle.

\section{REFERENCES}

1. World Health Organization. WHO global report on trends in prevalence of tobacco smoking, 2015. World
Health Organization; 2015. Accessed November 5, 2020. https://apps.who.int/iris/bitstream/ handle/10665/156262/9789241564922_eng.pdf

2. GBD 2015 Risk Factors Collaborators. Global, regional, and national comparative risk assessment of 79 behavioural, environmental and occupational, and metabolic risks or clusters of risks, 1990-2015: a systematic analysis for the Global Burden of Disease Study 2015. Lancet. 2016;388(10053):1659-1724. doi:10.1016/S0140-6736(16)31679-8

3. Spring B, Moller AC, Coons MJ. Multiple health behaviours: overview and implications. J Public Health (Oxf). 2012;34 (Suppl 1):i3-i10. doi:10.1093/pubmed/fdr111

4. Chiolero A, Wietlisbach V, Ruffieux C, Paccaud F, Cornuz J. Clustering of risk behaviors with cigarette consumption: A population-based survey. Prev Med. 2006;42(5):348353. doi:10.1016/j.ypmed.2006.01.011

5. European Commission. State of Health in the EU: Greece Country Health Profile 2019. OECD, European Observatory on Health Systems and Policies; 2020. Accessed April 20, 2021. https://ec.europa.eu/health/ sites/default/files/state/docs/2019_chp_gr_english.pdf

6. European Commission. Special Eurobarometer 429: Attitudes of Europeans towards tobacco and electronic cigarettes. European Union; 2015. Accessed July 10, 2020. http://ec.europa.eu/public_opinion/archives/ebs/ ebs_429_en.pdf

7. American Cancer Society. The Tobacco Atlas: Greece. Accessed May 7, 2021https://files.tobaccoatlas.org/wpcontent/uploads/pdf/greece-country-facts-en.pdf

8. Tsalapati K, Vardavas CI, Athanasakis K, et al. Going up in ashes? Smoking-attributable morbidity, hospital admissions and expenditure in Greece. Eur J Public Health. 2014;24(3):477-479. doi:10.1093/eurpub/cku040

9. Filippidis FT, Vardavas CI, Loukopoulou A, et al. Prevalence and determinants of tobacco use among adults in Greece: 4 year trends. Eur J Public Health. 2013;23(5):772-776. doi:10.1093/eurpub/cks148

10. Rachiotis G, Barbouni A, Katsioulis A, et al. Prevalence and determinants of current and secondhand smoking in Greece: results from the Global Adult Tobacco Survey (GATS) study. BMJ Open. 2017;7(1):e013150. doi:10.1136/bmjopen-2016-013150

11. Touloumi G, Karakatsani A, Karakosta A, et al. National Survey of Morbidity and Risk Factors (EMENO): Protocol for a Health Examination Survey Representative of the Adult Greek Population. JMIR Res Protoc. 2019;8(2):e10997. doi:10.2196/10997

12. Eurofound. Fourth European Working Conditions Survey (2005). Eurofound; 2005. Accessed May 10, 2021. https://www.eurofound.europa.eu/surveys/europeanworking-conditions-surveys/fourth-european-workingconditions-survey-2005

13. Craig CL, Marshall AL, Sjöström M, et al. International physical activity questionnaire: 12 -country reliability and 
validity. Med Sci Sports Exerc. 2003;35(8):1381-1395. doi:10.1249/01.MSS.0000078924.61453.FB

14. Papathanasiou G, Georgoudis G, Papandreou M, et al. Reliability measures of the short International Physical Activity Questionnaire (IPAQ) in Greek young adults. Hellenic J Cardiol. 2009;50(4):283-294. Accessed March 20, 2021. https://www.hellenicjcardiol.org/archive/full_ text/2009/4/2009_4_283.pdf

15. Schröder H, Fitó M, Estruch R, et al. A short screener is valid for assessing Mediterranean Diet adherence among older Spanish men and women. J Nutr. 2011;141(6):1140-1145. doi:10.3945/jn.110.135566

16. Hebestreit K, Yahiaoui-Doktor M, Engel C, et al. Validation of the German version of the Mediterranean Diet Adherence Screener (MEDAS) questionnaire. BMC Cancer. 2017;17(1):341. doi:10.1186/s12885-017-3337-y

17. Theodoridis X, Grammatikopoulou MG, Gkiouras K, et al. Food insecurity and Mediterranean diet adherence among Greek university students. Nutr Metab Cardiovasc Dis. 2018;28(5):477-485. doi:10.1016/j.numecd.2018.02.007

18. Eurostat. Tobacco consumption statistics. 2019. Accessed July 5, 2020. https://ec.europa.eu/eurostat/statisticsexplained $/$ index.php?title=Tobacco_consumption_ statistics

19. Amos A, Haglund M. From social taboo to "torch of freedom": the marketing of cigarettes to women. Tob Control. 2000;9(1):3-8. doi:10.1136/tc.9.1.3

20. Levine MD, Bush T, Magnusson B, Cheng Y, Chen X. Smoking-related weight concerns and obesity: differences among normal weight, overweight, and obese smokers using a telephone tobacco quit line. Nicotine Tob Res. 2013;15(6):1136-1140. doi:10.1093/ntr/nts226

21. De Vogli R, Santinello M. Unemployment and smoking: does psychosocial stress matter? Tob Control. 2005;14(6):389-395. doi:10.1136/tc.2004.010611

22. Prochaska JJ, Michalek AK, Brown-Johnson C, et al. Likelihood of Unemployed Smokers vs Nonsmokers Attaining Reemployment in a One-Year Observational Study. JAMA Intern Med. 2016;176(5):662-670. doi:10.1001/jamainternmed.2016.0772

23. Ramsey MW Jr, Chen-Sankey JC, Reese-Smith J, Choi K. Association between marital status and cigarette smoking: Variation by race and ethnicity. Prev Med. 2019;119:4851. doi:10.1016/j.ypmed.2018.12.010

24. Lopez AD, Collishaw NE, Piha T. A descriptive model of the cigarette epidemic in developed countries. Tob Control. 1994;3(3):242-247. doi:10.1136/tc.3.3.242

25. Huisman M, Kunst AE, Mackenbach JP. Educational inequalities in smoking among men and women aged 16 years and older in 11 European countries. Tob Control. 2005;14(2):106-113. doi:10.1136/tc.2004.008573

26. Martínez JA, Kearney JM, Kafatos A, Paquet S, Martínez-González MA. Variables independently associated with self-reported obesity in the European Union. Public Health Nutr. 1999;2(1A):125-133. doi:10.1017/s1368980099000178

27. Chiolero A, Faeh D, Paccaud F, Cornuz J. Consequences of smoking for body weight, body fat distribution, and insulin resistance. Am J Clin Nutr. 2008;87(4):801-809. doi:10.1093/ajen/87.4.801

28. Vardavas CI, Flouris AD, Tsatsakis A, Kafatos AG, Saris WH. Does adherence to the Mediterranean diet have a protective effect against active and passive smoking? Public Health. 2011;125(3):121-128. doi:10.1016/j.puhe.2010.11.012

29. Padrão P, Lunet N, Santos AC, Barros H. Smoking, alcohol, and dietary choices: evidence from the Portuguese National Health Survey. BMC Public Health. 2007;7(1):138. doi:10.1186/1471-2458-7-138

30. Falk DE, Yi HY, Hiller-Sturmhöfel S. An epidemiologic analysis of co-occurring alcohol and tobacco use and disorders: findings from the National Epidemiologic Survey on Alcohol and Related Conditions. Alcohol Res Health. 2006;29(3):162-171. Accessed March 20, 2021. https://www.ncbi.nlm.nih.gov/pmc/articles/ PMC6527037/pdf/162-171.pdf

31. Mammas IN, Bertsias GK, Linardakis M et al. Cigarette smoking, alcohol consumption, and serum lipid profile among medical students in Greece. Eur J Public Health. 2003;13(3):278-282. doi:10.1093/eurpub/13.3.278

32. World Health Organization. WHO Global NCD Action Plan 2013-2020: Appendix 3 update. World Health Organization; 2017. Accessed May 6, 2021. https://apps. who.int/iris/bitstream/handle/10665/259232/WHONMH-NVI-17.9-eng.pdf? sequence $=1 \&$ cisAllowed $=y$

33. Öberg, M, Jaakkola, M.S, Prüss-Üstün A, et al. 2010. Global estimate of the burden of disease from secondhand smoke / by Mattias Öberg ... [et al]. World Health Organization; 2010. Accessed March 20, 2021. https:// apps.who.int/iris/handle/10665/44426

34. Patrick DL, Cheadle A, Thompson DC, Diehr P, Koepsell T, Kinne S. The validity of self-reported smoking: a review and meta-analysis. Am J Public Health. 1994;84(7):10861093. doi:10.2105/ajph.84.7.1086

\section{CONFLICTS OF INTEREST}

The authors have each completed and submitted an ICMJE form for disclosure of potential conflicts of interest. The authors declare that they have no competing interests, financial or otherwise, related to the current work. G. Touloumi reports EU and National resources grants, as well as grants from the Hellenic Diabetes Association, all paid to her institution, related to the current work, and grants to her institution from Gilead Sciences Europe, UCL, ECDC, EU, University of Bristol, Harvard University, and National funds, outside the submitted work. A. Karakatsani reports funds from the European Union (European Social Fund) and national resources [grant number: MIS 376659], under the Operational Program "Education and Lifelong Learning". M. Gavana reports honoraria and support for expert testimony and attending meetings from MSD, Merck \& Co and Vianex SA respectively. 


\section{FUNDING}

The EMENO survey was implemented under the Operational Program 'Education and Lifelong Learning' and was co-funded by the European Union (European Social Fund) and national resources [Grant number: MIS 376659].

\section{ETHICAL APPROVAL AND INFORMED CONSENT}

The EMENO study was approved by the Ethics and Deontology Committee of the National and Kapodistrian University of Athens (Date: 8 November 2012, Protocol number: 1742) and by the Hellenic Data Protection Authority (Date: 7 December 2012, Protocol number: GN/EX/1069-1/07-122012). A modified version of the informed consent form (ICF) was approved by the Ethics and Deontology Committee of the National and Kapodistrian University of Athens (Date: 6 March 2013, Protocol number: 6315).

\section{DATA AVAILABILITY}

The data supporting this research are available from the authors on reasonable request.

\section{AUTHORS' CONTRIBUTIONS}

All authors contributed to the study conception, design and data collection. Material preparation was performed by AK and NK. Statistical and data analysis performed by NK and GT. The first draft of the manuscript was written by MG and reviewed-edited by AK and GT. All authors read and critically revised the final manuscript. The supervision of this study was by $\mathrm{AK}$ and GT.

PROVENANCE AND PEER REVIEW

Not commissioned; externally peer reviewed. 